PROCEEDINGS OF THE

AMERICAN MATHEMATICAL SOCIETY

Volume 131, Number 5, Pages 1619-1622

S 0002-9939(02)06916-2

Article electronically published on December 16, 2002

\title{
VAN DER WAERDEN SPACES AND HINDMAN SPACES ARE NOT THE SAME
}

\author{
MENACHEM KOJMAN AND SAHARON SHELAH \\ (Communicated by Alan Dow)
}

\begin{abstract}
A Hausdorff topological space $X$ is van der Waerden if for every sequence $\left(x_{n}\right)_{n \in \omega}$ in $X$ there is a converging subsequence $\left(x_{n}\right)_{n \in A}$ where $A \subseteq \omega$ contains arithmetic progressions of all finite lengths. A Hausdorff topological space $X$ is Hindman if for every sequence $\left(x_{n}\right)_{n \in \omega}$ in $X$ there is an IP-converging subsequence $\left(x_{n}\right)_{n \in F S(B)}$ for some infinite $B \subseteq \omega$.

We show that the continuum hypothesis implies the existence of a van der Waerden space which is not Hindman.
\end{abstract}

\section{INTRODUCTION}

A Hausdorff topological space $X$ is van der Waerden if for every sequence $\left(x_{n}\right)_{n \in \omega}$ in $X$ there is a converging subsequence $\left(x_{n}\right)_{n \in A}$ where $A \subseteq \omega$ contains arithmetic progressions of all finite lengths. A Hausdorff topological space $X$ is Hindman if for every sequence $\left(x_{n}\right)_{n \in \omega}$ in $X$ there is an IP-converging subsequence $\left(x_{n}\right)_{n \in F S(B)}$ for some infinite $B \subseteq \omega$. The term $F S(B)$ stands for the set of all finite sums (with no repetitions) over $B$ and IP-convergence to a point $x \in X$ means: for every neighborhood $U$ of $x$, there is some $n_{0}$ so that $\left\{x_{n}: n \in F S\left(B \backslash\left\{0,1, \ldots, n_{0}-1\right\}\right)\right\} \subseteq U$.

The classes of van der Waerden and of Hindman spaces were introduced in [2], [3] where it was shown that each class was productive and properly contained in the class of sequentially compact spaces, and that every Hausdorff space $X$ in which the closure of every countable set is compact and first countable is both van der Waerden and Hindman. The question was raised as to whether every Hausdorff space $X$ is van der Waerden if and only if it is Hindman. We answer this question in the negative using the Continuum Hypothesis.

1.1. Notation and combinatorial preliminaries. A set $A \subseteq \omega$ is an AP-set if it contains arithmetic progressions of all finite lengths. By van der Waerden's theorem [4], if an AP-set $A$ is partitioned into finitely many parts, at least one of

Received by the editors September 13, 2001 and, in revised form, December 12, 2001.

2000 Mathematics Subject Classification. Primary 54A20, 05A17, 03E35; Secondary 03E50.

The first author was partially supported by an Israel Science Foundation grant.

The second author was partially supported by an Israel Science Foundation grant. Number 782 in Shelah's list of publications.

The authors wish to acknowledge a substantial simplification made by the referee in the proof. The referee has eliminated an inessential use that the authors have made of the canonical van der Waerden theorem, all of whose known proofs use Szemerédi's theorem. 
the parts is AP. Let $\mathcal{I}_{A P}$ denote the collection of all subsets of $\omega$ which are not AP. $\mathcal{I}_{A P}$ is a proper ideal over $\omega$ and a set $A \subseteq \omega$ is AP if and only if $A \notin \mathcal{I}_{A P}$.

A set $A \subseteq \omega$ is an $I P$-set if there exists an infinite set $B \subseteq \omega$ so that $F S(B) \subseteq A$. $F S(B)=\left\{\sum F: F \subseteq A,|F|<\aleph_{0}\right\}$, where $\sum F$ stands for $\sum_{n \in F} n$. By Hindman's theorem [1], if an IP-set $A$ is partitioned into finitely many parts, at least one of the parts is IP. Let $\mathcal{I}_{I P}$ denote the collection of all subsets of $\omega$ which are not IP. $\mathcal{I}_{I P}$ is a proper ideal over $\omega$ and a set $A \subseteq \omega$ is IP if and only if $A \notin \mathcal{I}_{I P}$.

We shall need the following lemma which relates $\mathcal{I}_{A P}$ to $\mathcal{I}_{I P}$.

Lemma 1. Let $A$ be an $A P$ set and let $f: \omega \rightarrow \omega$. There exists an $A P$ set $C \subseteq A$ such that either

(1) $|f[C]|=1$ or

(2) $f$ is finite-to-one on $C$ and if $\left\langle x_{n}\right\rangle_{n=0}^{\infty}$ enumerates $f[C]$ in increasing order, then $\lim _{n \rightarrow \infty}\left(x_{n+1}-x_{n}\right)=\infty$.

In particular, $f[C] \in \mathcal{I}_{I P}$.

Proof. Suppose that for every AP set $C \subseteq A,|f[C]|>1$. We construct an AP set $C \subseteq A$ for which conclusion (2) holds.

For each $m \in \omega, A \cap f^{-1}[\{0,1, \ldots, m-1\}]$ is not an AP set because it is the finite union of sets on which $f$ is constant, and thus $A \backslash f^{-1}[\{0,1, \ldots, m-1\}]$ is an AP set. (Here we are using the fact that when an AP set is partitioned into finitely many parts, one of these parts is an AP set.)

We inductively construct sets $C_{n}$ for each $n \in \mathbb{N}$ such that

(a) for each $n \in \mathbb{N}, C_{n}$ is a length $n$ arithmetic progression and

(b) for all $n, m \in \mathbb{N}$, all $x \in C_{m}$, and all $y \in C_{n}$, if $m<n$, then $f(y) \geq f(x)+n$ and if $m=n$, then either $f(x)=f(y)$ or $|f(x)-f(y)| \geq n$.

Let $C_{1}$ be any singleton subset of $A$. Let $n \in \mathbb{N}$ and assume that we have chosen $C_{1}, C_{2}, \ldots, C_{n}$. Let $k=\max \bigcup_{i=1}^{n} f\left[C_{i}\right]$ and choose $i \in\{0,1, \ldots, n\}$ such that $\left(A \backslash f^{-1}[\{0,1, \ldots, k+n\}]\right) \cap f^{-1}[(n+1) \omega+i]$ is an AP set. Let $C_{n+1}$ be a length $n+1$ arithmetic progression contained in $\left(A \backslash f^{-1}[\{0,1, \ldots, k+n\}]\right) \cap f^{-1}[(n+1) \omega+i]$. Given $m \leq n+1, x \in C_{m}$, and $y \in C_{n+1}$, if $m \leq n$, then $f(x) \leq k$ and $f(y) \geq$ $k+n+1$, while if $m=n+1$, then either $f(x)=f(y)$ or $|f(x)-f(y)| \geq n+1$.

Let $C=\bigcup_{n=1}^{\infty} C_{n}$.

\section{THE SPACE}

Lemma 2. Assume $C H$. Then there exists a maximal almost disjoint family $\mathcal{A} \subseteq$ $\mathcal{I}_{I P}$ so that for every $A P$-set $B \subseteq \omega$ and every finite-to-one function $f: B \rightarrow \omega$ there exists an $A P$-set $C \subseteq B$ and $A \in \mathcal{A}$ so that $f[C] \subseteq A$.

Proof. We construct from $\mathrm{CH}$ an almost disjoint family $\mathcal{A}=\left\{A_{\alpha}: \alpha<\omega_{1}\right\} \subseteq \mathcal{I}_{I P}$ by induction on $\alpha$. The enumeration $\left\{A_{\alpha}: \alpha<\omega_{1}\right\}$ may contain repetitions. Let $\left\{A_{n}: n<\omega\right\} \subseteq \mathcal{I}_{I P}$ be a collection of infinite and pairwise disjoint sets.

Fix a list $\left\langle\left(f_{\alpha}, B_{\alpha}\right): \omega \leq \alpha<\omega_{1}\right\rangle$ of all pairs $(f, B)$ in which $B \subseteq \omega$ is an AP-set and $f: B \rightarrow \omega$ is a finite-to-one function.

Suppose $\omega \leq \alpha<\omega_{1}$ and that $A_{\beta}$ has been chosen for all $\beta<\alpha$. Consider the pair $\left(f_{\alpha}, B_{\alpha}\right)$. If there exists a finite set $\left\{\beta_{0}, \beta_{1}, \ldots, \beta_{\ell}\right\} \subseteq \alpha$ so that $f_{\alpha}^{-1}\left[\bigcup_{i \leq \ell} A_{\beta_{i}}\right]$ is $\mathrm{AP}$, let $A_{\alpha}=A_{0}$. 
Otherwise, enumerate $\alpha$ as $\left\langle\beta_{i}: i<\omega\right\rangle$, and now for all $n<\omega$ the set $f_{\alpha}^{-1}\left[\bigcup_{i<n} A_{\beta_{i}}\right]$ is not AP, hence $B_{\alpha} \backslash f_{\alpha}^{-1}\left[\bigcup_{i<n} A_{\beta_{i}}\right]$ is AP. Let an arithmetic progression $D_{n} \subseteq B_{\alpha} \backslash f_{\alpha}^{-1}\left[\bigcup_{i<n} A_{\beta_{i}}\right]$ of length $n$ be chosen for all $n$. Then $B^{\prime}:=$ $\bigcup_{n \in \omega} D_{n}$ is an AP-subset of $B_{\alpha}, f_{\alpha}\left[B^{\prime}\right]$ is infinite (because $f_{\alpha}$ is finite-to-one) and $\left|f_{\alpha}\left[B^{\prime}\right] \cap A_{\beta}\right|<\aleph_{0}$ for all $\beta<\alpha$. By Lemma 1 find an AP-set $B^{\prime \prime} \subseteq B^{\prime}$, so that $f_{\alpha}\left[B^{\prime \prime}\right] \in \mathcal{I}_{I P}$, and define $A_{\alpha}=f_{\alpha}\left[B^{\prime \prime}\right]$.

The family $\mathcal{A}=\left\{A_{\alpha}: \alpha<\omega_{1}\right\}$ is clearly an almost disjoint family of (infinite) sets, and $\mathcal{A} \subseteq \mathcal{I}_{I P}$.

Suppose now that $B \subseteq \omega$ is an AP-set and that $f: B \rightarrow \omega$ is finite-to-one. There is an index $\omega \leq \alpha<\omega_{1}$ for which $(B, f)=\left(B_{\alpha}, f_{\alpha}\right)$. At stage $\alpha$ of the construction of $\mathcal{A}$, either $f^{-1}\left[A_{\beta_{0}} \cup \cdots \cup A_{\beta_{\ell}}\right]$ was AP for some finite set $\left\{\beta_{0}, \ldots, \beta_{\ell}\right\} \subseteq \alpha$, hence $f^{-1}\left[A_{\beta}\right]$ was AP for some single $\beta<\alpha$, or else $f^{-1}\left[A_{\alpha}\right]$ was AP. In either case, there is an AP-set $C \subseteq B$ and $A \in \mathcal{A}$ so that $f[C] \subseteq A$.

Finally, to verify that $\mathcal{A}$ is maximal let an infinite set $D \subseteq \omega$ be given and let $f: \omega \rightarrow D$ be the increasing enumeration of $D$. Since there is an AP-set $C \subseteq \omega$ and $A \in \mathcal{A}$ so that $f[C] \subseteq A$, it is clear that $D \cap A$ is infinite.

Theorem 3. Suppose CH holds. Then there exists a compact, separable van der Waerden space which is not Hindman.

Proof. Let $\mathcal{A}$ be as stated in Lemma 2 For each $A \in \mathcal{A}$ let $p_{A} \notin \omega$ be a distinct point. Define a topology $\tau$ on $Y=\omega \cup\left\{p_{A}: A \in \mathcal{A}\right\}$ by requiring that $Z \in \tau$ if and only if for all $p_{A} \in Z$ the set $A \backslash Z$ is finite. Then for each $A \in \mathcal{A}, A \cup\left\{p_{A}\right\}$ is a compact neighborhood of $p_{A}$, so $\tau$ is a locally compact Hausdorff topology in which $\omega$ is a dense and discrete subspace. Let $X=Y \cup\{p\}$ be the one-point compactification of $\tau$.

It was shown in [3, Theorem 10] that when $\mathcal{A} \subseteq \mathcal{I}_{I P}$ is maximal almost disjoint, the space constructed in this way is sequentially compact but not Hindman. To keep this paper self-contained, we repeat the simple argument showing that $X$ is not Hindman. For each $n \in \omega$, let $x_{n}=n$ and suppose we have some infinite $B \subseteq \omega$ such that $\left(x_{n}\right)_{n \in F S(B)}$ IP-converges to $q \in X$. Then $q \notin \omega$. If $q=p_{A}$ for some $A \in \mathcal{A}$, then $A$ is an IP set. So $q=p$. By the maximality of $\mathcal{A}$, pick $A \in \mathcal{A}$ such that $A \cap B$ is infinite. But then $X \backslash\left(A \cup\left\{p_{A}\right\}\right)$ is a neighborhood of $p$ and for no $n$ does one have $F S(B \backslash\{0,1, \ldots, n-1\}) \subseteq X \backslash\left(A \cup\left\{p_{A}\right\}\right)$.

We have yet to see that $X$ is van der Waerden. Suppose $f: \omega \rightarrow X$ is given. Let $g: f[\omega] \rightarrow \omega$ be 1-1. By Lemma 1 we can find an AP set $B \subseteq \omega$ so that $(g \circ f) \mid B$ is constant or finite-to-one, and hence $f\lceil B$ is constant or finite-to-one. In the former case, the sequence $(f(n))_{n \in B}$ is constant, and therefore converges. So assume that $f \nmid B$ is finite-to-one. Since either $f^{-1}[\omega] \cap B$ or $B \backslash f^{-1}[\omega]$ is AP, we may assume, by shrinking $B$ to some AP-subset, that either $f[B] \subseteq \omega$ or $f[B] \subseteq X \backslash(\omega \cup\{p\})$.

In the former case, there is some $A \in \mathcal{A}$ and AP-set $C \subseteq B$ so that $f[C] \subseteq A$. Since $f\left\lceil B\right.$ is finite-to-one, $(f(n))_{n \in C}$ converges to $p_{A}$. In the latter case, we claim that the sequence $(f(n))_{n \in B}$ converges to $p$. To see this, let $Z$ be a compact subset of $Y$, so that $X \backslash Z$ is a basic neighborhood of $p$. Then $Z \backslash \omega$ is finite so, since $f \nmid B$ is finite-to-one, $(f(n))_{n \in B}$ is eventually in $X \backslash Z$.

\section{REFERENCES}

[1] N. Hindman. Finite sums from sequences within cells of a partition of N . J. Comb. Theory (Series A), 17:1-11, 1974. MR 50:2067 
[2] M. Kojman. Van der Waerden spaces. Proc. Amer. Math. Soc., 130:631-635, 2002. MR 2002i:54018

[3] M. Kojman. Hindman spaces. Proc. Amer. Math. Soc., 130:1597-1602, 2002.

[4] B. L. van der Waerden. Beweis eine Baudetschen Vermutung Nieuw Arch. Wisk., 15:212-216, 1927.

Department of Mathematics, Ben Gurion University of the Negev, Beer Sheva, Israel E-mail address: kojman@cs.bgu.ac.il

Institute of Mathematics, The Hebrew University of Jerusalem, Jerusalem, Israel E-mail address: shelah@ma.huji.ac.il 